\title{
The effect of neoprene thumb abduction splints on upper limb function in children with cerebral palsy
}

\author{
Ashleigh Hughes, BSc OT (UCT), MSc OT (Wits) \\ Private Practitioner, Kloof, Kwazulu Natal; Postgraduate Student, Department of Occupational Therapy, School of Therapeutic \\ Sciences, Faculty of Health Sciences University of the Witwatersrand
}

\section{Denise Franzsen, BSc (Wits), MSc OT (Wits) PhD (Wits) DHT(UP)}

Senior Lecturer, Department of Occupational Therapy, School of Therapeutic Sciences, Faculty of Health Sciences University of Witwatersrand

\section{Juliana Freeme, BSc (Wits), MSc OT (Wits)}

Tutor, Department of Occupational Therapy, School of Therapeutic Sciences, Faculty of Health Sciences University of Witwatersrand

Introduction: Children with cerebral palsy (CP) have limitations in hand function due to weakness and spasticity, with long term effects causing shortening and stiffness of muscles in the hand and arm. The soft neoprene thumb abductor splint is prescribed by occupational therapists to correct these impairments. There is insufficient evidence for the effectiveness of this splint on upper limb function in children with CP with a thumb-in-palm deformity within the South African public health context.

Methods: A non-blinded randomised intervention study was carried out in a CP clinic at a tertiary level public hospital. Twenty-eight CP children between the age of 18 and 68 months, presenting with thumb-in-palm deformity, functioning on a Level II to IV on the Gross Motor Function Classification Scale (GMFCS) participated in the study.

The Quality of Upper Extremity Skills Test (QUEST) was used to assess upper limb function in terms of dissociated movement, grasps, weight bearing and protective extension to obtain baseline information. Both the intervention and control groups received monthly individual occupational therapy and a home programme over a three month period. The intervention group additionally received a soft neoprene thumb abduction splint.

Results: No statistically significant differences between the intervention and control groups were found. Clinically relevant between group differences indicated by effect sizes were found however with large effect sizes the for the total QUEST score and weight bearing.

The effect size for improvement within the intervention group was large for dissociated movement and weight bearing with and without the splint on as well as for grasps with the splint on. Only dissociated movement showed a clinically relevant medium effect size for change within the control group. Protective extension decreased within both groups except for the intervention group with the splint on.

The caregivers/parents perceived the home programme as easy to follow, and those who were prescribed the splint mostly reported that it was easy to use.

Conclusion: Soft neoprene thumb abduction splints have a positive effect on weight bearing and upper limb function in children with thumb-in-palm deformity, particularly with the splint on. Further research is recommended in the area of splinting in CP.

Key words: Cerebral palsy, thumb-in-palm deformity, neoprene thumb abductor splint, QUEST

\section{INTRODUCTION}

Skilled use of the hands and upper limbs in activities enables interaction with the environment, and is thus essential for participation in the occupational performance areas of self-care, work or education, and leisure'. At least $50 \%$ of children with cerebral palsy (CP) have limitations in arm and hand use, which is the main cause of their dysfunction in occupational performance areas ${ }^{2}$. The hand movements of a child with CP can be slow and weak, with incoordination, incomplete finger dissociation, spasticity, and impaired tactile sensation ${ }^{3}$. Other impairments described in the literature include increased deep tendon reflexes, tremors and muscular hypertonicity. In the upper limb, spasticity presents in a flexor pattern, which includes spasticity of the flexors and adductors of the fingers and thumb. The spasticity develops into hypertonicity over time, and muscles of the wrist and hand are often shortened and stiff in a fisted position ${ }^{4}$. Deformities, due to the shortening and stiffness of muscles of the first web space, and hypermobility of the metacarpophalangeal and distal interphalangeal joints of the thumb are therefore common in children with $\mathrm{CP}^{5}$. The thumb-in-palm deformity is caused by a permanent position of thumb adduction and flexion, which affects both pinches and grasps required for normal hand function ${ }^{6}$. Intervention to correct this deformity and to support the hand and wrist in a functional position is essential to allow children with $\mathrm{CP}$ an opportunity to use their hands more functionally.

Occupational therapists use splinting as one of the interventions to rehabilitate the above-mentioned impairments and to improve the use of the hands. Hand splints are specifically prescribed for children with $\mathrm{CP}$ to stabilise and protect the joints, stretch and lengthen muscles, prevent contractures or to maintain range of motion. They 
may be categorised as 'non-functional hand splints' that stabilise and provide static positioning of the hand or upper limb, or 'functional hand splints' prescribed to facilitate the position of the upper limb for active movement during activity participation. Functional hand splints include soft neoprene splints, recommended for their ease of use, as well as the comfort compared to a hard splint. An example of a neoprene splint specifically for the use of a thumb-inpalm deformity is the soft neoprene thumb abduction splint. This splint allows for better hand function and grasps, by maintaining the thumb in an abducted or unopposed position. The splint is made from a neoprene material providing neutral warmth to the hand ${ }^{7,8}$ as well as a prolonged stretch of the adductor muscles of the thumb, both of which have a temporary inhibitory effect on spasticity'. The soft neoprene thumb abductor splint does not restrict active movement of the thumb in grasp and release. During active movement it maintains full range of passive motion, preventing shortening of muscle fibres and facilitating remodelling of connective tissue around the muscle ${ }^{8,10}$. Thus, movement of the hand is facilitated while stretching the intrinsic muscles, meaning that the splint can be worn during functional activities ${ }^{7,11}$.

Previous research has shown that splinting, in conjunction with therapy, is more effective than therapy alone, and that splinting may benefit some children with $\mathrm{CP}$ with regards to the quality of movement in the short-term ${ }^{7}$. However, it is important to determine what effect this improvement in quality of movement has on their ability to function in their everyday lives, as conflicting results have been reported in other studies ${ }^{3,8,12,13}$. The findings also emphasised that there is little evidence for the continued effect achieved by splinting, and that it has been found that once splinting is removed, the benefits ceased ${ }^{7}$. Splinting material and regular monitoring of the correct fit and condition of the splint - if required for a long period of time - can be a costly intervention ${ }^{12}$. Therefore, the achievement of outcomes in the long term through the use of splinting must be weighed up against the cost of this intervention method used over an extended period ${ }^{7}$.

A study by Goodman and Bazyk ${ }^{14}$ on the effectiveness of soft functional hand splints prescribed to children with CP indicated that wearing a short thumb opponens splint for six hours a day over a four-week period, improved range of motion, dexterity and quality of movement, but had no effect on strength. In a later study, Ten Berge et $\mathrm{al}^{8}$ showed that there was improved hand function while the neoprene thumb opponens splint was worn over an eightweek period in children with unilateral spastic CP attending weekly therapy sessions. However the effect of splinting on occupational performance goals, and carryover when the splint was removed, was not as clear.

The effect of splinting in facilitating hand function and the improvement of movement of the entire upper limb was not reported on in these studies, therefore the long term benefits were not discussed. The findings also highlighted that in view of the high cost, appearance of the splint, and long-term commitment required to the adherence of wearing a splint, clinicians need to be sure that the use of this adjunctive technique is justified ${ }^{7}$.

Therefore, although there have been previous studies on the soft neoprene thumb abduction splint, there is a lack of information regarding the effect of the splint on upper limb function. Whether the splint has a long-term effect on the use of the hand in children with $\mathrm{CP}$ who attend monthly therapy sessions, and whether the economic impact in the long term is justified compared to the amount of improvement observed.

The aims of the current study were therefore twofold. Firstly, it was to determine the effect on upper limb function in children with $\mathrm{CP}$ with a thumb-in-palm deformity involved in monthly individual therapy and a home programme, who were prescribed a soft neoprene thumb abductor splint over a three month period. Secondly, the study aimed to gather feedback from all the parents/caregivers regarding the use of the home programmes, and feedback regarding the splint prescribed to the participants.

\section{METHODS}

A non-blinded randomised intervention study was conducted at a clinic at a large public hospital in South Africa where occupational therapists, physiotherapists and speech therapists provide a oncea-month service for children with CP. An experimental design was used to determine the effect of a soft neoprene thumb abductor splint over a three month period, compared to upper limb function in children with $\mathrm{CP}$ with a thumb-in-palm deformity who did not receive the splint.

Ethical clearance was obtained from the Human Research Ethics Committee (Medical) at the University of the Witwatersrand (MIOI 020) and permission was obtained from the Gauteng Department of Health, the CEO of the hospital where the clinic was run and the rehabilitation clinical manager.

\section{Study sample}

Children with CP aged between 18 months to 6 years, with thumbin-palm deformity and who were on level II to VI on the Gross Motor Function Classification Scale (GMFCS) ${ }^{15,16}$ and attending the clinic at least once every second month were identified by the researcher and other treating therapists. The researcher was one of the occupational therapists providing a service at the clinic during the time of the study. The researcher obtained the names and contact details of these identified children, and invited the parents/ caregivers of the children to participate in the study by giving them an information letter and explaining the study to them. Informed consent was obtained from parents or caregivers of 40 children to participate in the study.

Children with a co-morbid or previous diagnosis of hydrocephalus, tuberculosis, meningitis, or acute illness were excluded, and these conditions were noted in the medical records. Children with a static contracture of the thumb adductors and/or wrist were also excluded, and this was determined through a range of motion evaluation by the researcher or treating therapist. Due to the nature of the study, it was important for the homogeneity of the sample to include children who were otherwise healthy. Improvement of hand function could be affected by illnesses, such as those stated above, and could potentially affect the muscle strength and tone in the hand, which could compromise the results of the study.

After the exclusion criteria were applied to the 40 children who had consented to participate and who were eligible, 28 participants were included in the study. The participants were randomly assigned on a I:I ratio by a rehabilitation team member, thus not the researcher, to either the control or intervention group according to a computer-generated random allocation table. There was no specific allocation to the different groups according to age or type of $\mathrm{CP}$ in the participants.

\section{Measuring instruments}

The Gross Motor Function Classification Scale (GMFCS) The GMFCS levels ${ }^{15,16}$ were used to indicate whether the children with $C P$ attending the clinic could be included in the study. Therapists at the clinic where the study was conducted routinely use the GMFCS to classify all children with CP who utilise the services. Therefore, all children included in the study had already been evaluated through the use of the tool, and they were functioning on level II to VI of the scale. Children with gross motor functioning on these specified levels can walk, but with limitations, with or without a hand-held mobility device. They may also be dependent on the use of a wheelchair, which they can self-propelled manually, or by using a powered device, or they may be propelled by someone ${ }^{15,16}$.

\section{The Quality of Upper Extremity Skills Test (QUEST)}

The QUEST was developed by DeMatteo et al in 1993 and it measures the components of hand function as well as the quality of upper limb movement in children with neuromotor dysfunction $^{17}$. The test includes four aspects of hand function: dissociated movements, grasps, weight bearing and protective extension. This 
standardised assessment is easily accessible via the internet and can be purchased on https://canchild.ca/en/resources/49-quality-ofupper-extremity-skills-test-quest, and no extra training is required in order to administer the test. The QUEST score is sensitive to disability rather than age, and is used with children ranging from 18 months to eight years. The assessment has been tested for use in children with CP and has been found to have good inter-observer reliability $(0.96)$, good observer reliability, $(0.5 \mathrm{I}-0.94)$, and good test-retest reliability $(0.75-0.95)^{17}$. The QUEST has also been compared to the Peabody Developmental Motor Scales (PDMSFM) ${ }^{18}$, and Melbourne Assessment of Unilateral Upper Limb Function (MUUL) ${ }^{19}$ both of which have excellent internal consistency, test-retest reliability, and inter- and intra-rater reliability. Construct, content, criterion and evaluative validity was high with a correlation of 0.84 between the QUEST and PDMS-FM ${ }^{18}$ and a correlation of 0.83 between the QUEST and MUUL ${ }^{19}$. The QUEST has also been reported to be responsive to change over time i $^{17,20}$

The equipment required to administer the test comprises everyday objects which are used routinely in therapy rooms. The therapist observes the child's movement during set activities which are done seated at a table or on a mat on the floor ${ }^{17}$. The scale took on average 30 minutes to complete per child, and consists of the following domains and items:-

In the dissociated movement domain, movement is observed while the child is reaching for objects such as a puppet or blocks. The therapist observes how much range of movement the child has in the shoulders (flexion, extension, and abduction), the elbow (flexion and extension), the forearm (supination and pronation) and the wrist (flexion and extension). There are 19 items with sections for movement of the shoulder, elbow, wrist, finger, and grasp and release of a one inch cube.

In the grasps domain, observations are made while the child picks up objects such as blocks, crayons and cereal off the table or mat and the type of grasp is scored (palmar, digital pronate, tripod, pincer, radial palmer). The therapist also observes and records the child's ability to release objects. This section has 15 items including sitting posture during grasps, grasping a one inch cube, cereal, and a pencil or crayon.

The weight bearing domain items are performed in either prone or four-point kneeling. The child may not have any equipment assisting them to maintain the position (e.g. a wedge or roller). The position of the hand and arm is then scored (elbow flexed or extended, hand open or closed). This section includes five items, including position in prone or four-point, without and then with reach, and weight bearing in a sitting position.

Protective extension is evaluated in the last domain, and it evaluates the child's ability to prevent themselves from falling when their weight is displaced. This is done in cross-legged or ring-sitting, and again considers whether the elbow is extended or flexed and whether the hand is open or closed. This section has three items, including forward, side and backward protective extension.

The examiner also rates the child's hand function between 0 (poor) and good (10). Poor hand function is described as minimal independent hand grasps with no active release and the inability to combine grasping and releasing, and good hand function is described as spontaneous reach grasp and release with good eye-hand coordination.

Lastly, the examiner rates the child's spasticity as "none", "mild", "moderate" or "severe". The level of spasticity describes how much the spasticity interferes with spontaneous movement if associated movement is present, how stiff the muscles are and if the increase in tone is present at rest.

The examiner also comments on whether the child cooperates during the assessment.

Each item is scored as 'yes' (tick) or 'no' (cross) depending on whether or not the child can complete the movement or not. If the item cannot be tested then it is marked as 'NT' (not tested). In order to calculate the total score of the test, the ticks, crosses and NT's are summed for each section and a total is calculated us- ing the four scores from the four sections, by using the prescribed formula to obtain the raw score. The score is then converted to a standardised score from zero to $100^{17}$. Since this is a criterion referenced test, each child's score is used to establish a baseline and then to identify if any improvement has occurred.

\section{The pre-study parent/caregiver questionnaire}

This questionnaire was completed by the main caregivers of all the children who were included in the study to determine the context in which they resided, the needs of the caregiver in relation to explaining the home programme and the time they had available to complete the home programme as well as the time and finances they had to bring the child for monthly therapy. The questionnaire was developed by the researcher and it was completed at the commencement of the study. The questionnaire included questions regarding their relationship to the child, age, gender, race, language, highest level of education, employment status and occupation. Questions were also answered regarding the area where the child resides, the housing type and circumstances, income of the household, and type of income. Information regarding the child was obtained as well, including age, gender, consultations by medical professionals, and diagnosis and the caregiver's insight into the child's diagnosis.

\section{The post-study parent/caregiver questionnaire}

A post-study questionnaire was issued to caregivers in the experimental and control groups which allowed caregivers to comment on any difficulties they had with carrying out the splinting regime or the home programme. The questionnaire was designed by the researcher with the view to obtaining information about factors which may have influenced wearing of the splint or carrying out the home programme. The questions were open-ended and allowed the researcher to gather information concerning the participant's splint which may have affected the overall outcome of the intervention.

\section{Procedure}

The inclusion and exclusion criteria were applied, and the researcher assessed all the participating children using the QUEST at the beginning of the study. All parents/caregivers completed the pre-study questionnaire with the assistance of a translator or the researcher.

All the participants received individual therapy on a monthly basis from the researcher and received an individualised and updated home programme at each appointment. Parents/caregivers were individually trained in the application of the home programmes and the use of the splint.

The home programmes were designed by the researcher for the purpose of the research study. It was based on the routine and standard home programme which attendees of the $\mathrm{CP}$ clinic receive from the therapists. The researcher made improvements to the routine home programme by translating it into Zulu (the language spoken mostly by the people who live in the surrounding areas of the clinic), adding simple diagrammes to enhance understanding, and by adding active exercises and functional tasks to the routine stretches. The home programmes generally consisted of three parts. Firstly, the parents/caregivers would perform basic neurodevelopmental theory (NDT) preparation techniques which consisted of trunk rotation which then lead into rolling and reaching in later programmes as well as shoulder mobilisation ${ }^{21}$ to normalise tone in the trunk and upper limb. Secondly active graded exercises of the upper limb were covered, starting with proximal joints and moving to more distal joints such as the wrist and fingers. It was recommended by the researcher that the caregivers allowed the child to initiate the movement and move as independently as possible. Only when the child could not move any further would the caregiver facilitate the rest of the movement with the child ${ }^{21}$. The last part of each home programme contained skills which could be incorporated into daily tasks to increase participation in activities of daily living (ADL) and to facilitate functional skills. Caregivers carry out all ADL for the 
children and they are often not given the opportunity to try themselves. The researcher felt it was important to include functional activities so that the children were given opportunities to attempt basic ADL and show their caregivers that they are capable of doing some activities themselves or with minimal assistance. When a child is actively engaged in the activity, functional activities capture the attention of the child and allow them to engage in the activity which promotes their learning ${ }^{22}$. Manipulation of objects with different sizes, shapes and textures encourages interaction of visual and somatosensory information which increases attention and increases learning ${ }^{22,23}$. Therefore, different ADL activities were included, using appropriate objects such as a cloth for wiping their mouth and holding their bottle or cup. The caregivers were expected to perform the home programme with the child three times daily in the morning, midday and evening.

Participants in the intervention group were individually fitted with a standard neoprene thumb abduction splint ${ }^{8}$ by the researcher and prescribed additional information on how to incorporate the splint into the home programme. The splint was made with soft neoprene fabric and fitted by the researcher, as described in literature $^{8}$. It fits on the thumb, in the web space and around the palmar and dorsal surfaces, and secured on the ulnar aspect of the hand ${ }^{14}$ The researcher found the splints could be made in a short period of time and that they were durable, lasting for at least three months.

The splints were reviewed at every appointment and adjusted where necessary. Caregivers were also expected to complete an adherence diary for the duration of the study on a daily basis, and they documented their attendance to therapy, number of hours the splint was worn, and whether the home programme was performed as prescribed.

The QUEST was administered again at the three-month appointment. During the appointment the final assessment for the intervention group was administered twice, once while they were wearing the splint and again with the splint removed. This was done to determine if the splint had a direct effect on the change in hand function and quality of movement of the upper limb. The researcher was therefore aware of which participants were in the intervention group, and this prevented blinding in the study.

The post-intervention parent/caregiver questionnaire was completed at the end of the intervention period by the caregivers in the control and intervention groups.

There was a loss to follow up of nine children (32\%) with five children in the intervention group and four children in the control group not returning for the final assessment. This resulted in a final sample size of 19 children. The reason given for non-attendance was a lack of finance for transport, as some participants were required to travel long distances to the clinic.

\section{Data Analysis}

An intention to treat analysis was used which included the data from all 28 children in the initial assessment and 19 children in the final assessment. Data for the mean change from initial and final QUEST domain and total scores within the control and intervention groups were determined using the nonparametric Wilcoxon sign rank test since the data were ordinal. The differences between the initial and final scores were also calculated for the intervention group with and without the splint. The Cohen $\mathrm{d}$ effect sizes were determined for differences from the initial to the final assessment means for each groups' scores to establish the magnitude of the treatment effect within each group. A small effect size ranges from $0.20-0.49$, a medium effect size $0.50-0.79$ and a large effect size is $\geq 0.80$.

The significance of difference in the change from the initial to final mean QUEST domain and total scores was compared for the control and intervention groups using the nonparametric Mann-Whitney $U$ test due to the ordinal nature of the data. The
Cohen $d$ effect sizes were also used to measure the magnitude of the treatment effect when comparing the mean change in scores between the two groups.

Statistical analysis was performed using the statistical package STATA 12 Statistical Package ${ }^{24}$. Confidence intervals were set at $95 \%$, and testing was done at the 0.05 level of significance.

\section{RESULTS}

\section{Demographics}

The ages of the child participants ranged from 18 to 68 months with more boys (I7/28) than girls (II/28). All but one of the primary caregivers, were the mothers of the children. The level of education of the caregivers varied with the majority of caregivers having I 2 years of schooling or less. Most caregivers (19/28) were unemployed. The household income of two thirds of the participants families were between R500 - R5000 a month.

There was no significant difference in terms of age, language spoken, level of education and employment status of caregivers between the control and intervention groups. The child participants in the two groups were also comparable, as there was no statistically significant difference between the two groups in terms of age and gender.

\section{Change in QUEST scores from initial to final assessment within the control and intervention groups}

There was an improvement seen in the control group from initial to final assessments for all domains and the total QUEST scores, except for protective extension for which the score decreased with a negative effect size (-0.14). No statistically significant differences were found between the initial and final QUEST domain and total scores. The greatest improvement was recorded between the initial and final dissociated movement scores, with a medium effect size (0.72). The grasps and weight-bearing components showed slight improvement with small effect sizes of 0.03 and 0.01 respectively, and the total QUEST score also had a small effect size of 0.1 (Table I).

Table I: Initial $(n=14)$ and final $(n=10)$ scores for the control group

\begin{tabular}{|l|c|c|c|c|}
\hline & $\begin{array}{c}\text { Initial assessments } \\
\text { Mean (SD) } \\
\mathbf{n}=~ 14\end{array}$ & $\begin{array}{c}\text { Final assessments } \\
\text { Mean (SD) } \\
\mathbf{n}=10\end{array}$ & $\mathbf{p}$ value & $\begin{array}{c}\text { Cohen d } \\
\text { Effect size } \\
\text { (ES) }\end{array}$ \\
\hline $\begin{array}{l}\text { Dissociated } \\
\text { movements }\end{array}$ & $46.48(12.64)$ & $55.63(12.13)$ & 0.74 & 0.72 \\
\hline Grasps & $11.90(20.35)$ & $12.25(11.6 I)$ & 0.88 & 0.03 \\
\hline $\begin{array}{l}\text { Weight } \\
\text { Bearing }\end{array}$ & $45.56(19.36)$ & $45.80(17.05)$ & 0.11 & 0.01 \\
\hline $\begin{array}{l}\text { Protective } \\
\text { Extension }\end{array}$ & $51.5 \mathrm{I}(25.50)$ & $48.8 I(18.90)$ & 0.11 & -0.14 \\
\hline Total & $38.26(15.42)$ & $39.95(20.92)$ & 0.12 & 0.10 \\
\hline
\end{tabular}

In the intervention group, an improvement between the initial and final scores for all domains and total QUEST were found, except for protective extension which showed a decrease in effect size $(-0.0$ I $)$ when assessed with the splint removed. No statistically significant differences were found between the initial and final QUEST domain and total scores. With the splint on, the improvement was greater than without the splint for all domains and total QUEST scores, except for the weight bearing component where the final score without the splint was higher. The effect sizes for dissociated movement, grasp and weight bearing were large (I.0I, 0.82 and 0.85 respectively), as was the mean change in the total QUEST score (1.28) with the splint on.

Only dissociated movement and weight bearing had large effect sizes ( 0.87 and 0.95 respectively) with the splint removed. A similar 
Table II: Initial $(n=14)$ and final $(n=9)$ scores for the intervention group

\begin{tabular}{|c|c|c|c|c|}
\hline & $\begin{array}{c}\text { Initial assessments Mean } \\
\text { (SD) } \\
n=14\end{array}$ & $\begin{array}{l}\text { Final assessments Mean } \\
\qquad \begin{array}{c}\text { (SD) } \\
\mathbf{n}=9\end{array}\end{array}$ & p value & $\begin{array}{l}\text { Cohen d } \\
\text { Effect size } \\
\text { (ES) }\end{array}$ \\
\hline Dissociated movements * & $44.34(11.76)$ & $53.12(17.39)$ & 0.48 & $0.87^{* * * *}$ \\
\hline Dissociated Movements ** & & $56.25(14.69)$ & & I.0I*** \\
\hline Difference without and with splint & & $3.12(9.17)$ & & \\
\hline Grasps* & $3.72(11.00)$ & $8.35(10.62)$ & 0.37 & 0.42 \\
\hline Grasps** & & $11.11(6.93)$ & & $0.82 * * *$ \\
\hline Difference without and with splint & & $2.76(6.22)$ & & \\
\hline Weight Bearing* & $4 I .66(14.31)$ & $60.44(24.88)$ & 0.60 & 0.95 \\
\hline Weight Bearing** & & $56.60(20.55)$ & & $0.85 * * *$ \\
\hline Difference without and with splint & & $-3.84(15.36)$ & & \\
\hline Protective Extension* & $51.25(16.67)$ & $48.33(20.75)$ & 0.26 & -0.01 \\
\hline Protective Extension** & & $56.66(24.58)$ & & 0.32 \\
\hline Difference without and with splint & & $8.33(/ 4.43)$ & & \\
\hline Total* & $33.26(9.32)$ & $40.35(13.73)$ & 0.13 & 0.52 \\
\hline Total** & & $45.16(13.57)$ & & $1.28 * * *$ \\
\hline Difference without and with splint & & $2.59(3.15)$ & & \\
\hline
\end{tabular}

medium effect size was found for the total QUEST for the mean change without the splint $(0.52)$. (Table II)

\section{Change in QUEST scores between control and intervention groups}

There was an overall improvement in the mean scores for all domains and the total QUEST score for both groups. The improvement in the intervention group was greater both with and without the splint on, than that in the control group. Only the change in the mean scores for dissociated movements and for protective extension without the splint indicated less improvement in the intervention group than for the control group over the three month period. The effect sizes were small however at -0.06 and -0.0 I respectively. Even with the splint on, the effect size of the change in mean scores between the control and experimental groups was small for dissociated movements $(0.46)$ and protective extension (0.45).

The weight-bearing domain and the total QUEST score were found to have improvement in the intervention group that resulted in large effect sizes when compared to the control group. The effect size between the intervention and control groups for change in mean scores for weight bearing without the splint was I.53 and with splint on was I.22. None of the mean changes in the groups were statistically significant (Table III).
A large effect size was found for the total QUEST score $(0.82)$ when the participants were assessed with the splint on and a small effect size of 0.43 without the splint when the mean change in the two groups was compared. (Figure I on page 8)

\section{Feedback from parents/caregivers on the use of home programmes}

Most caregivers (I8/19) reported that the home programme was easy to follow. All the caregivers of the intervention group stated that the splint was easy to put on and take off although two of the nine caregivers experienced difficulties with the splint at some point in the study. All the caregivers of the child participants who were evaluated for the final assessment felt that there was some improvement in their child over the duration of the study, also reporting improvement in upper limb functioning in the child's ability to do everyday activities.

\section{DISCUSSION}

The study aimed to determine the effect of a soft neoprene thumb abductor splint on the upper limb function in children with $\mathrm{CP}$ with thumb-in-palm deformity. The participants were randomly allocated to the control or intervention groups, where both groups received monthly therapy with an individualised home programme, but the participants in the intervention group also received the

Table III: Comparison of change from initial to final assessment mean scores for control and intervention groups $(n=19)$

\begin{tabular}{|c|c|c|c|c|c|c|}
\hline & \multirow{2}{*}{$\begin{array}{c}\begin{array}{c}\text { Control Group } \\
\mathbf{n}=10\end{array} \\
\text { Mean Change scores }\end{array}$} & \multicolumn{4}{|c|}{$\begin{array}{l}\text { Intervention group } \\
\qquad \mathrm{n}=9\end{array}$} & \multirow[t]{2}{*}{ P value } \\
\hline & & $\begin{array}{c}\text { Mean Change scores } \\
\text { No splint }\end{array}$ & $\begin{array}{l}\text { Cohen d } \\
\text { Effect size }\end{array}$ & $\begin{array}{c}\text { Mean Change scores } \\
\text { Splint on }\end{array}$ & Cohen d Effect size & \\
\hline $\begin{array}{l}\text { Dissociated } \\
\text { movements }\end{array}$ & 9.15 & 8.78 & -0.06 & 11.91 & 0.46 & 0.53 \\
\hline Grasps & 0.35 & 4.63 & 0.21 & 7.39 & 0.34 & 0.43 \\
\hline Weight Bearing & 0.24 & 18.78 & I.53*** & 14.94 & I.22*** & 0.40 \\
\hline $\begin{array}{l}\text { Protective } \\
\text { Extension }\end{array}$ & -2.7 & -2.92 & -0.01 & 5.41 & 0.45 & 0.91 \\
\hline Total & 1.69 & 7.09 & 0.43 & 11.9 & $0.82 * * *$ & 0.96 \\
\hline
\end{tabular}




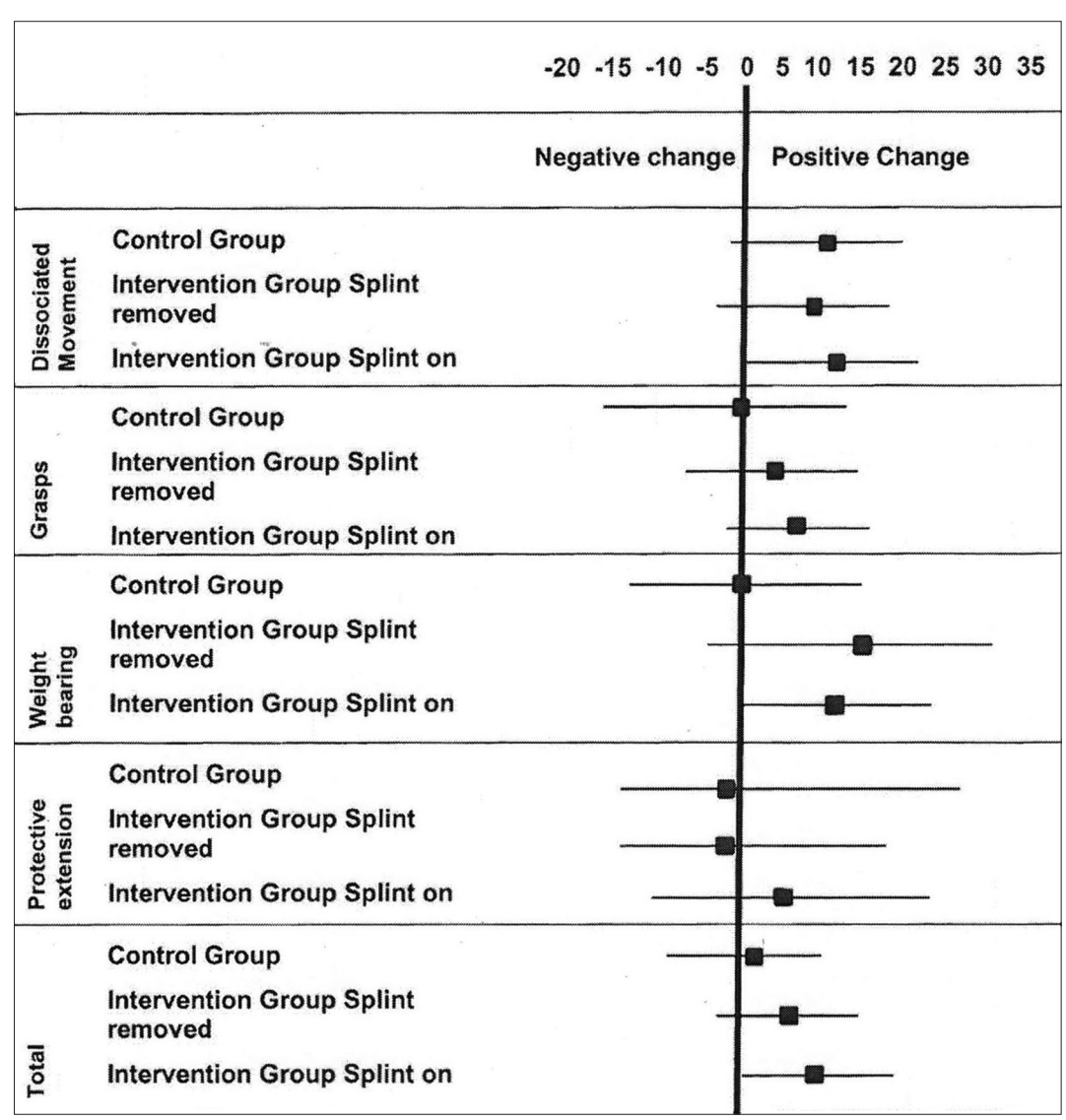

Figure I: Mean score change on the QUEST scores for the control group and intervention group (with and without the splint)

soft neoprene thumb abductor splint. Upper limb functioning was measured using the QUEST, which included a total score, and scores for dissociated movements, grasps, weight bearing and protective extension domains. The intervention group was evaluated during the final assessment with and without the splint on. The effect size results in this sample of children with CP indicate that the soft neoprene thumb abductor splint had a positive clinical effect with large effective sizes for some domains, on upper limb function, although the improvement was not statistically significant.

When considering within group changes the participants in the small to medium effect sizes indicated some improvement in upper limb functioning, especially for dissociated movement in the control group after three months of intervention and implementing a home programme (Table I). These results indicated less effective change than that indicated by the medium to large effect sizes found for improvement over the three month intervention period for the participants in the intervention group while wearing of the splint and executing the home programmes. Protective extension scores decreased for the control group and the intervention group when not wearing the splint (Table II).

All domains and the total QUEST scores showed an increase when the initial and final scores of the intervention group with the splint on, were compared. The final assessment score for weightbearing however, was higher without the splint than with the splint on. There was a large effect size for change in scores when the participants wore the splint for dissociated movements, grasp, weight bearing and the total QUEST scores (Table II).

When comparing the effect size for the change in the mean scores of the control group and intervention group (assessed with the splint on), all effect sizes for the domains and total QUEST scores were positive except one, meaning that although both groups improved, the improvement was greater in the intervention group when tested with the splint on and off, except for protective extension with the splint removed. The total QUEST score with the splint on, showed the most improvement with a large effect size when compared to the control group, indicating that participants in the intervention group scored at the $79^{\text {th }}$ percentile of the control group (Table III). This dropped to the $66^{\text {th }}$ percentile with the splint removed and the small effect size found.

Each of the domains assessed by the QUEST need to be considered separately as the results differed. The dissociated movements subtest evaluated the child's ability to reach and then grasp and release a block ${ }^{17}$. When the participants in the intervention group were assessed with their splints on, the size effect for dissociated movement was greater than without the splint, however the control group showed greater improvement than the intervention group without the splint on (Table III). It appears that change in the movement in the shoulders, elbow, forearm and wrist and fingers achieved in this study may have been due to the NDT home programme with the splint having more of an effect on the hand and fingers where the ability to grasp and release the block, was scored. Wearing the splint allowed participants to achieve grasp and release of a large object more effectively, as the splint allowed for better hand function, by maintaining the thumb in an abducted or unopposed position ${ }^{7}$.

The type of grasp the child used differed with the splint on as they tended to use a more functional grasp, such as a radial palmer or digital pronate grasp when wearing the splint as opposed to a palmer or palmer supinate grasp, thus improving their score for the section. The grasp sub-test includes pinch and pencil grasp, and with the splint on, the participants were able to grasp a greater variety of objects with more functional grasps. This demonstrates that the splint was effective when used in conjunction with activities to maintain a functional position of the thumb out of the palm ${ }^{3}$.

Although the change in grasp in the intervention group was large with splint on, when the change in the intervention and control groups were compared this indicated little difference between the groups with a small effect size, both with and without the splint on. There was less carryover in grasping when the splint was removed in the intervention group, although the change was greater than that found for the control group. Therapy and the home programme appeared to have little effect on the improvement of grasp in this sample and the home programme needed to be combined with a splint to achieve change in this domain. This study supports earlier findings that soft splinting of the thumb has some positive effects, especially when grasping objects in the QUEST test items with the splint on, but as with other studies, in the short three month period this effect was not maintained when the splint was removed ${ }^{8,14}$.

Since protective extension is a postural reaction, the lack of change and slight decrease in the scores for this aspect were therefore not unexpected. Although the subtest evaluated the position of the hand, in a similar way to the weight bearing subtest, protective extension required quick displacement out of their base of support ${ }^{17}$ which did not allow the child any time to adjust the position of their hand. In a child with CP righting reactions often take longer to initiate than in a normal developing child, therefore the vestibular system will be activated during this time and spasticity increases. The flexed position of the upper limb, slow righting reactions and an increase in spasticity therefore make it difficult for the child to extend their arms appropriately to prevent falling ${ }^{25}$. The slight improvement found in the intervention group with the splint on may be due to the thumb being positioned out of the palm and the hand being less fisted when the reflex was elicited.

Weight-bearing had a large effect size when comparing the control and intervention groups, both with and without the splint on indicating that the splint had a positive effect for this component of upper limb function even when the split was removed. Weight 
bearing requires extension of the elbow and wrist, with fingers abducted and extended to form a base to lean on. Weight-bearing becomes challenging for children with $\mathrm{CP}$, because spasticity in the upper limb will present in a flexor pattern, which includes spasticity of the flexors and adductors of the fingers and thumb. The spasticity develops into hypertonicity over time, and muscles of the wrist and hand are often shortened and stiff in a fisted position ${ }^{4}$. The soft neoprene thumb abductor splint allows for better opening of the hand by maintaining the thumb in an abducted or unopposed position allowing the child to support themselves on an open hand when weight bearing ${ }^{7,26}$. Therefore, with a more functional hand position weight-bearing can occur, which accounts for the improvement in this domain score. Similar results were found by Deshkar et al. ${ }^{27}$ using a splint especially designed to position the hand for weight bearing on the upper limb. They also found that the lengthening and strengthening of the muscles in the hand resulted in an improvement of weight-bearing in children with $\mathrm{CP}$.

The improvement in domain and total QUEST scores in the control group (Table I), indicated that the therapy and home programme provide some successful results on their own, although improvement was greater with the prescription of the splint in conjunction with the home programme (Table III).

When considering the caregivers/parents feedback regarding the home and splinting programmes, this was mostly positive as the home programme was suited to their needs and they found it easy to follow. Those who used the splint also felt it was easy to use. They also reported that they observed improvement in their children's functioning, even without having insight into the QUEST scores, for both control and intervention groups.

Since the participants were from low socioeconomic circumstances with their caregivers/parents having no more than secondary schooling, and being mostly unemployed, studies into the cost effectiveness of the splint for children from this South African context are needed to justify the cost of this type of splint. This study shows that the use of this splint appears to provide greater benefits in upper limb functioning than traditional interventions and home programmes on their own, with caregivers/parents being positive about its use in conjunction with a home programme for children that can only attend therapy monthly, if that often.

Limitations of the study included the loss to follow up of nine participants, which resulted in the small sample of children participating in the study. The reasons for nonattendance are those commonly reported for patients who rely on outpatient care at public hospitals in South Africa ${ }^{28}$. The findings should also be interpreted with caution when applying to the general population, as the sample was not homogenous due to the wide variety in ages and types of $C P$.

\section{CONCLUSION}

The study established a positive effect on upper limb function in children with $\mathrm{CP}$ with a thumb-in-palm deformity, involved in monthly individual therapy and a home programme over a threemonth period when an intervention group who were prescribed a soft neoprene thumb abductor splint, were compared to a control group who were not prescribed a splint. There were no significant differences between the initial and final scores of either the control and intervention groups, or between the initial and final scores of each group, although there was improvement in the upper limb function of both groups. The effect size of the mean changes in scores in total QUEST scores for the intervention group with the splint on was large, and greater than the control group, indicating that the soft neoprene thumb abductor splint does have a positive clinical effect on upper limb functioning. This change in the intervention group was not as large without the splint on, indicating loss of carry over once the splint was removed.

While protective extension showed some deterioration over the three months, the participants in the intervention group did achieve some benefit with better positioning of the thumb and the a less fisted hand, when the reflex was elicited while wearing the splint. The large and medium positive effect size for the change in dissociated movements and grasps with the splint on found in the intervention group where not clinically relevant when compared to the improvement in the control group. Thus, due to the fact that this study showed that the soft neoprene thumb abductor splint is useful during dissociated movement and grasps only when it is worn, and not better for dissociated movement than therapy and a home programme when not worn, further studies should be conducted to indicate the longer term and permanent effects of the splint in this population. This also suggests that the participants should continue to wear their neoprene thumb abductor splints while doing functional activities, especially for the improvement of grasp as there is little carryover when the neoprene thumb abductor splint is removed at this stage. Weight bearing on the upper limb achieved the greatest benefit from the wearing of a splint and carryover of this aspect was positive and appears to have been retained in upper limb function once the splint is removed.

Feedback from all the parents/caregivers regarding the use of the home programmes, and the splint prescribed to the participants in the intervention group was positive and the home programmes and splints were well received by the participants. More in-depth research is required to explore reasons for compliance to home programmes and splint wearing, and the cost effectiveness of the soft neoprene thumb abductor splint in children with CP from low socio-economic contexts attending occupational services at public hospitals in South Africa.

\section{ACKNOWLEDGEMENTS}

The Faculty of Health Sciences at the University of Witwatersrand for the Research Endowment Grant made available for the purchase of splinting material for this study.

\section{CONFLICT OF INTEREST STATEMENT}

The authors declare that there is no conflict of interests.

\section{REFERENCES}

I. Exner C. Development of Hand Skills. In: Case-Smith J, (ed.). Occupational Therapy for Children. Missouri: Elsevier Inc, 2005.

2. Fedrizzi E, Pagliano E, Andreucci E and Oleari G. Hand function in children with hemiplegic cerebral palsy: prospective follow-up and functional outcome in adolescence. Developmental Medicine and Child Neurology. 2003; 45: 85-91.

3. Burtner P and Poole J. Therapeutic intervention as the Body Structure and Function Level to Support Children's Upper Extremity Function. In: Eliasson A-C and Burtner P, (eds.). Improving Hand Function in Children with Cerebral Palsy: theory, evidence and intervention. Londonn: Mac Keith Press; 2008: 230-259.

4. Krigger K. Cerebral Palsy: An overview. American Family Physician. 2006; 73: 91-100.

5. Vogtle L. Upper Extermity Intervention in Cerebral Palsy: A neurodevelopmental approach. In: Henderson A and Pehoski C, (eds.). Hand Function in the Child Foundations and Remediation. St Louis: Mosby; 2006: 345-368.

6. Tonkin MF, A, Koman A and Leclercq C. Surgical Management of Thumb Deformity in Cerebral Palsy. Surgical Management of Thumb Deformity in Cerebral Palsy; 2008.

7. Jackman M, Novak I and Lanin N. Effectiveness of hand splints in children with cerebral palsy: A systematic review with meta-analysis. Develop Medicine and Child Neurology. 2014; 56: I38-I47.

8. Ten Berge S, Boonstra A, Dijkstra I P, Hadders-Algra M, Haga N and Maathuis $C$. A systematic evaluation of the effect of thumb opponens splints on hand function in children with unilateral spastic cerebral palsy. Clinical Rehabilitation. 2012; 26: 362-37I.

9. Gabriel LS. Pediatric Splinting. Introduction to Splinting: A Clinical Reasoning and Problem Solving Approach. Missouri: Elsevier Mosby; 2008.

10. Wilton J. Casting, splinting, and physical and occupational therapy of hand deformity and dysfunction in cerebral palsy. Hand Clinics. 2003; 19: 573 - 584.

II. Aaron DH. Pediatric Hand Therapy. In: Henderson A and Pehoski C, (eds.). Hand Function in the Child Foundations for Remediation. 
Missouri: Mosby Elsevier; 2006: 403-434.

12. Autti-Rämö I, Suoranta J, Anttila H, Malmivaara A and Mäkelä $A$. Effectiveness of Upper and Lower Limb Casting and Orthoses in Children with Cerebral Palsy. An Overview of Review Articles. American Journal of Physical Medicine \& Rehabilitation. 2006; 85: 89-103.

13. Teplicky R, Russel D and Law M. Casts, Splint and Orthoses - Upper Extremity Review of Effectiveness Literature for Children with Neurological Disorders. CanChild; 2003.

14. Goodman G and Bazyk S. The Effects of a Short Thumb Opponens Splint on Hand Function in Cerebral Palsy: A Single-Subject Study. American Journal of Occupational Therapy. 199I; 45: 726-73I.

15. Alshryda S and Wright J. Development and Reliability of a System to Classify Gross Motor Function in Children with Cerebral Palsy. Classic Papers in Orthopaedics. Springer; 2014: 575-577.

16. Palisano R, Rosenbaum P, Walter S, Russell D, Wood E and Galuppi B. Development and reliability of a system to classify gross motor function in children with cerebral palsy. Developmental Medicine and Child Neurology. 1997; 39: 214-223.

17. DeMatteo C, Law M, Russel D, Pollock N, Rosenbaum P and Walter S. The Reliability and Validity of the Quality of Upper Extremity Skills Test. Physical and Occupational Therapy in Pediatrics. 1993; 13: I-I8.

18. Folio MR and Fewell RR. Peabody developmental motor scales. Pro-ed; 2000.

19. Bourke-Taylor H. Melbourne assessment of unilateral upper limb function: construct validity and correlation with the pediatric evaluation of disability inventory. Developmental medicine and child neurology. 2003; 45: 92-96.

20. Corn K, Imms C, Timewell G, Carter C, Collins L, Dubbeld S et al. Impact of Second Skin Lycra Splinting on the Quality of Upper Limb Movement in Children. British Journal of Occupational Therapy. 2003; 66: 464-472.

21. Humphry R and Case-Smith J. Working with Families. In: Case-Smith J, (ed.). Occupational Therapy for Children. Missouri: Elsevier Inc; 2005: II7-159.

22. Raine $S$. The current theoretical assumptions of the Bobath concept as determined by the members of BBTA. Physiotherapy Theory and Practice. 2007; 23: 137-I52.

23. Law M, Missiuna C, Pollock N and Stewart D. Foundations for Occupational Therapy Practice with Children. In: Case-Smith J, (ed.). Occupational Therapy for Children. Missouri: Elsevier Inc, 2005.

24. Carr J and Shepherd R. Reaching and manipulation. Neurological Rehabilitation Optimizing Motor Performance. China: Churchill Livingstone Elsevier; 20II: I23-I62.

25. StataCorp. STATA I2. College Station, Texas. 2012.

26. Case-Smith J. Hand Skill Development in the Context of Infants' Play: Birth to 2 years. In: Henderson A and Pehoski C, (eds.). Hand Function in the Child: Foundations for Remediation. Misssouri: Mosby Elsevier: 2006: II7-142.

27. Pontèn E. Muscle Alterations Due to Spasticity. Improving Hand Function in Children with Cerebral Palsy: theory, evidence and intervention. London: Mac Keith Press; 2008.

28. Deshkar A, Naik S and Deshkar A. Management of tone and hand functions in cerebral palsy: Inhibitive weight bearing splint as an adjunct modality. Journal of Evolution of Medical and Dental Sciences. 2014; 3: 9173-9178.

29. Ntamo N, Buso D and Longo-Mbenza B. Factors affecting poor attendance for outpatient physiotherapy by patients discharged from Mthatha general hospital with a stroke. South African Journal of Physiotherapy. 2013; 69: 13-18. 